\title{
Major Contribution of GREB1L Alterations to Severe Inner Ear Malformation Largely in a Non-mendelian Fashion
}

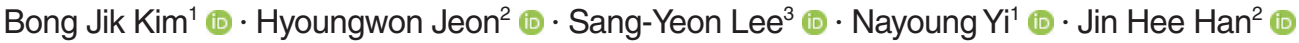 \\ Go Hun $\mathrm{Seo}^{4}$ iD $\cdot$ Seung-Ha Oh ${ }^{3}$ id $\cdot$ Byung Yoon $\mathrm{Choi}^{2}$ (D) \\ ${ }^{1}$ Department of Otolaryngology-Head and Neck Surgery, Chungnam National University Sejong Hospital, Chungnam National University \\ College of Medicine, Sejong; ${ }^{2}$ Department of Otorhinolaryngology-Head and Neck Surgery, Seoul National University Bundang Hospital, Seoul \\ National University College of Medicine, Seongnam; ${ }^{3}$ Department of Otorhinolaryngology-Head and Neck Surgery, Seoul National University \\ Hospital, Seoul National University College of Medicine, Seoul; ${ }^{4} 3$ billion Inc., Seoul, Korea
}

Severe inner ear malformation (IEM), including common cavity (CC) or cochlear aplasia with dilated vestibule (CADV), is challenging in terms of auditory rehabilitation and genetic counseling [1]. Little is known regarding its genetic etiologies, although occasional reports have suggested involvement of GREB1L (growth regulation by estrogen in breast cancer 1-like) gene [2,3]. Alterations of GREB1L, a neural crest regulatory molecule, have been reported to cause kidney anomalies [4]. Interestingly, most pedigrees showed maternal transmission, leading to hypotheses of genomic imprinting or effects on male fertility [4]. Four variants of GREB1L have been reported as candidate variants for profound sensorineural hearing loss under various inheritance modes (DFNA80 [MIM: \#619274]): de novo, autosomal dominant with or without reduced penetrance [3]. However, the genetic etiology and mode of inheritance of severe IEM remain largely unknown. Through this study, we suggest that GREB1L alterations are the major etiology of CC/CADV, and they manifest the phenotype largely in a non-Mendelian fashion. Our results point towards the novel concept that severe IEM could develop due to autosomal genetic alterations, but frequently in a non-Mendelian fashion.

\footnotetext{
- Received October 6, 2021

Revised November 8, 2021

Accepted November 20, 2021

- Corresponding author: Byung Yoon Choi

Department of Otorhinolaryngology-Head and Neck Surgery, Seoul

National University Bundang Hospital, Seoul National University College of Medicine, 82 Gumi-ro 173beon-gil, Bundang-gu, Seongnam 13620, Korea

Tel: +82-31-787-7406, Fax:+82-31-787-4057

E-mail: choiby@snubh.org
}

Five unrelated nonsyndromic hearing loss families (SNUBHCADV/CC cohort) with severe IEM on both sides and CADV/CC on at least one side were recruited from 2012 to 2019 at Seoul National University Bundang Hospital. Five probands of the five pedigrees all showed profound deafness requiring bilateral cochlear implants (CIs) for appropriate auditory rehabilitation. Their audiologic and radiologic data were rigorously reviewed. During the same period, 421 CI recipients, including 220 pediatric subjects, were also ascertained to have severe to profound hearing loss at the same hospital. The 215 pediatric CI recipients other than the five patients recruited here had conditions including enlarged vestibular aqueduct (EVA, $n=36)$, incomplete partition type 1 (IP-1, $n=3)$, and IP-3 ( $n=3)$.

Exome sequencing (ES) was performed for 150 of the 220 pediatric $\mathrm{CI}$ recipients. $\mathrm{ES}$ data were not available for 70 patients. In detail, some patients with EVA $(n=36)$ and IP-3 $(n=3)$ were directly sequenced for SLC26A4 and POU3F4, while a subset of subjects with nonsyndromic hearing loss and deafness $(n=11)$ carrying GJB2 variants were genetically diagnosed after a screening panel was performed, and some participants at the beginning of the study $(n=20)$ underwent panel sequencing instead of ES. Family-based trio ES was performed in four of the five CADV/CC families (SB120, SB259, SB282, and SH169). ES followed by bioinformatics analysis narrowed down the candidate variants $[5,6]$. The pathogenic variants were classified according to the American College of Medical Genetics and Genomics (ACMG) guideline and the recently specified ACMG/Association for Molecular Pathology (AMP) hearing loss rules [7,8]. Pedigrees, audiograms, and abnormal radiologic findings from the five CADV/CC families are displayed in Fig. 1.

Copyright $\odot 2022$ by Korean Society of Otorhinolaryngology-Head and Neck Surgery.

This is an open-access article distributed under the terms of the Creative Commons Attribution Non-Commercial License (https://creativecommons.org/licenses/by-nc/4.0) which permits unrestricted non-commercial use, distribution, and reproduction in any medium, provided the original work is properly cited. 


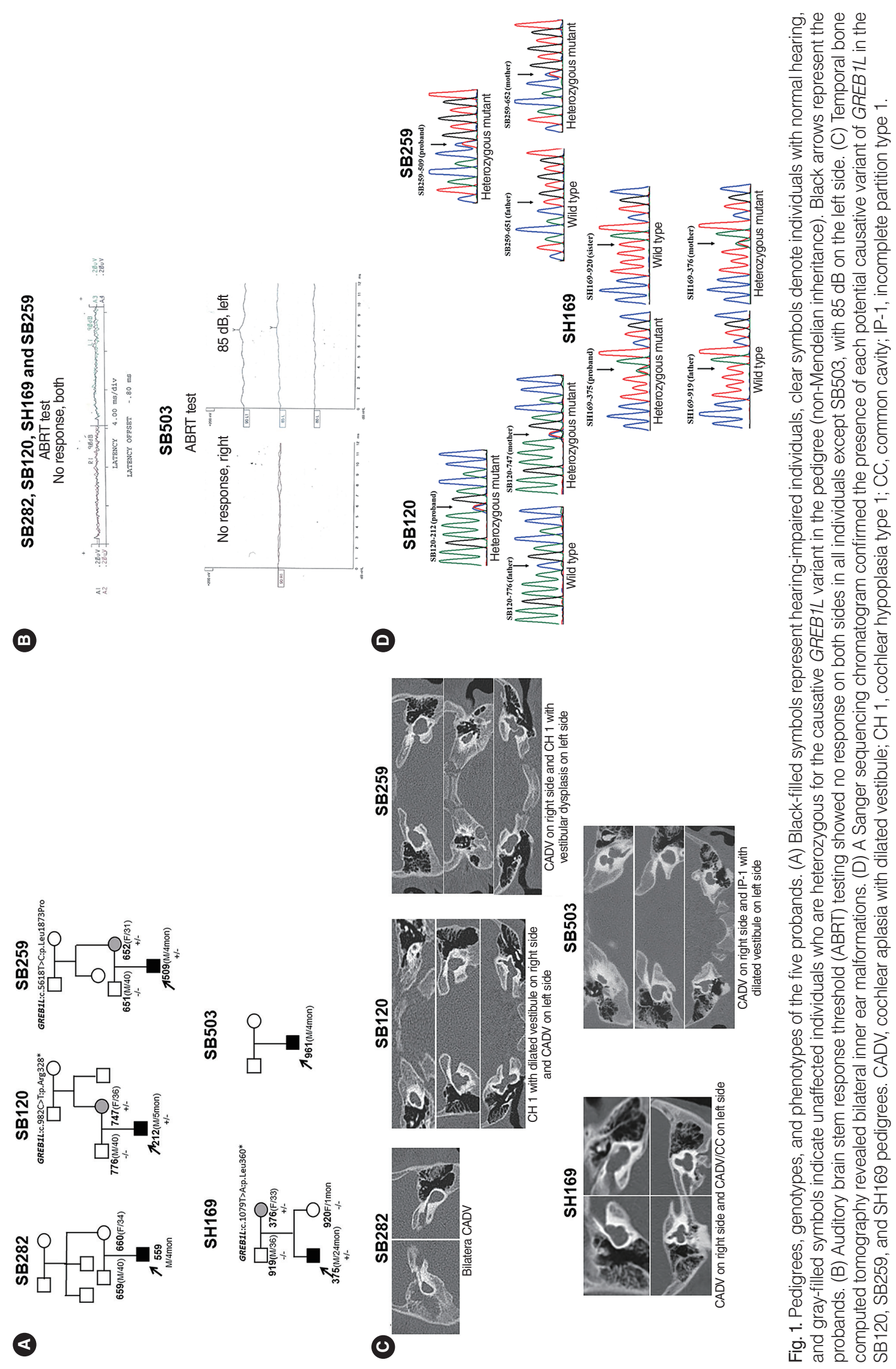


All steps in this study were approved by the Institutional Review Board of Seoul National University Bundang Hospital (IRBB-1007-105-402). Written informed consent was obtained from all individuals or their guardians (for minors).

The SNUBH-CADV/CC cohort constituted 1.19\% (5/421) of all CI recipients and $2.27 \%(5 / 220)$ of pediatric recipients. We identified three heterozygous variants of GREB1L, including one novel missense variant (c.5618T $>C$ ) and two nonsense variants (one novel) (c.982C $>\mathrm{T}$ and c.1079T $>\mathrm{A}$, novel) from three CADV/CC families (SB259, SB120, and SH169), while the genetic etiology was not determined in two CADV/CC families (SB282 and SB503) (Fig. 1, Table 1). Public databases including Global minor allele frequency and Korean Reference Genome Database and in silico studies including Rare Exome Variant Ensemble Learner and Combined Annotation Dependent Depletion further demonstrated the pathogenic potential of three heterozygous variants of the GREB1L gene, which were classified as pathogenic (c.982C $>\mathrm{T})$, likely pathogenic (c.1079T $>\mathrm{A})$ and VUS (c.5618T $>$ C), respectively, according to the ACMG/AMP guidelines (Table 1). Each candidate variant was confirmed to be present through Sanger sequencing.

No convincing GREB1L variants were detected in any of the other 145 pediatric CI recipients with available ES data, giving rise to a statistically significant predilection of GREB1L variants exclusively in the CADV/CC cohort (Fisher's exact test, $P<0.001$ ). Noticeably, all three families showed discordant segregation between GREB1L variants and CADV/CC among family members. Specifically, all probands carrying GREB1L variants were boys who always inherited the GREB1L variant from their normalhearing mothers (Fig. 1).

Our study showed a statistically significant, and most likely causal, relationship between GREB1L variants and CADV/CC, since GREB1L variants were exclusively detected in CADV/CC subjects. A significant genetic load of GREB1L variants in CADV/ $\mathrm{CC}$ was also suggested by the detection rate of $60 \%$ in the SNUBHCADV/CC cohort.

Of particular note, the segregation of GREB1L variants in all three families did not conform to conventional Mendelian inheritance. Our observations are consistent with the previous detection of a GREB1L variant descended from a normal-hearing mother [3] and the proposed pathogenesis of kidney agenesis caused by GREB1L variants [4]. Intrafamilial variability might exist, or this finding could be potentially related to the fact that GREB1L is an androgen-regulated gene [9]. However, the carrier mothers in the cohort showed no overt hearing or renal phenotypes. Thus, genomic imprinting of GREB1L with preferential expression of the maternal mutant allele (silencing of the paternal allele) could be a possibility for further research. GREB1L alterations should be suspected as a major genetic contributor to severe IEMs, potentially through a non-Mendelian inheritance pattern.

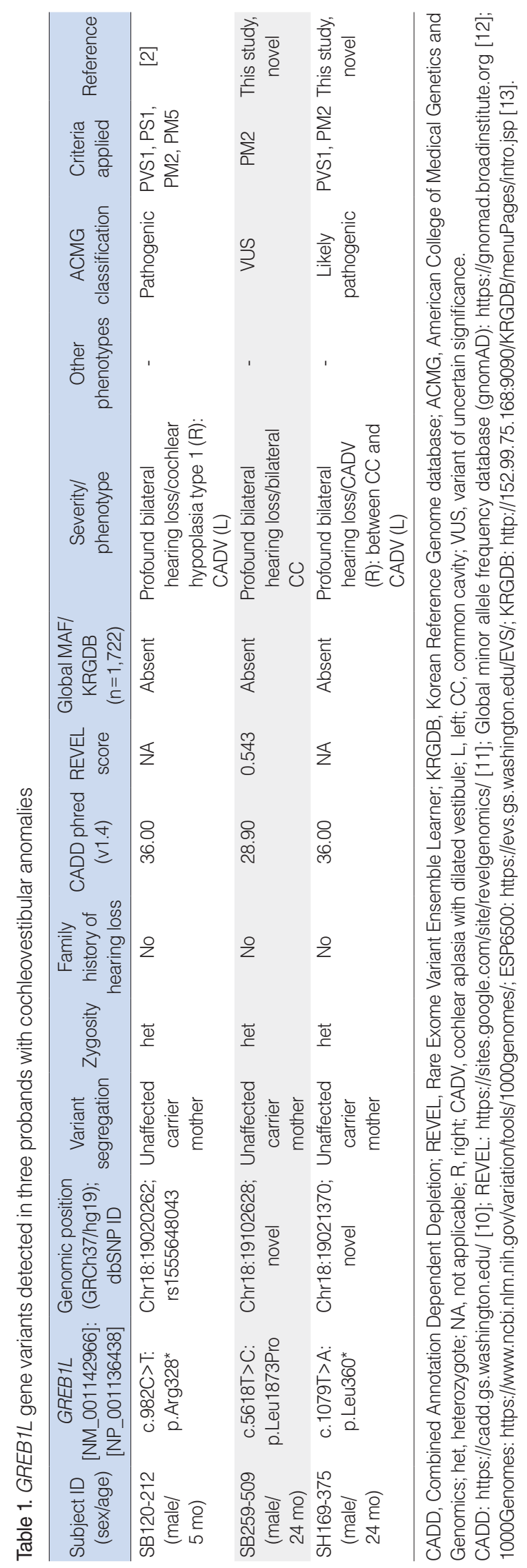




\section{CONFLICT OF INTEREST}

No potential conflict of interest relevant to this article was reported.

\section{ACKNOWLEDGMENTS}

This study was supported by the SNUBH Intramural Research fund (SNUBH 16-2019-0006 to BYC) and a grant from the Korean Society of Medical Genetics and Genomics (KSMG-AR202001 to BJK).

\section{ORCID}

Bong Jik Kim https://orcid.org/0000-0002-6384-2171

Hyoungwon Jeon https://orcid.org/0000-0002-8235-7649

Sang-Yeon Lee

Nayoung Yi

https://orcid.org/0000-0003-3566-8708

Jin Hee Han

https://orcid.org/0000-0001-7688-0313

Go Hun Seo

https://orcid.org/0000-0001-5810-5161

Seung-Ha Oh

https://orcid.org/0000-0003-1518-1791

https://orcid.org/0000-0003-1284-5070

Byung Yoon Choi https://orcid.org/0000-0001-5125-2118

\section{AUTHOR CONTRIBUTIONS}

Conceptualization: CBY, BJK. Data curation: HJ, NY, JHH, SYL. Formal analysis: JHH, NY, GHS. Funding acquisition: CBY, BJK. Methodology: GHS. Project administration: JHH. Visualization: HJ.Writing-original draft: BJK. Writing-review \& editing: SHO, CBY.

\section{REFERENCES}

1. Sennaroğlu L, Bajin MD. Classification and current management of inner ear malformations. Balkan Med J. 2017 Sep;34(5):397-411.

2. Schrauwen I, Kari E, Mattox J, Llaci L, Smeeton J, Naymik M, et al. De novo variants in GREB1L areassociated with non-syndromic inner ear malformations and deafness. Hum Genet. 2018 Jul;137(6-7): 459-70.

3. Schrauwen I, Liaqat K, Schatteman I, Bharadwaj T, Nasir A, Acharya A, et al. Autosomal dominantly inherited GREB1L variants in individuals with profound sensorineural hearing impairment. Genes (Basel). 2020 Jun;11(6):687.

4. DeTomasi L, David P, Humbert C, Silbermann F,Arrondel C,Tores F, et al. Mutations in GREB1L cause bilateral kidney agenesis in humans and mice.Am J Hum Genet. 2017 Nov;101(5):803-14.

5. Kim BJ, Oh DY, Han JH, Oh J, Kim MY, Park HR, et al. Significant Mendelian genetic contribution to pediatric mild-to-moderate hearing loss and its comprehensive diagnostic approach. Genet Med. 2020 Jun;22(6):1119-28.

6. Kim BJ, Kim AR, Lee C, Kim SY, Kim NK, Chang MY, et al. Discovery of $\mathrm{CDH} 23$ as a significant contributor to progressive postlingual sensorineural hearing loss in Koreans. PLoS One. 2016 Oct;11(10): $\mathrm{e} 0165680$.

7. Richards S, Aziz N, Bale S, Bick D, Das S, Gastier-Foster J, et al. Standards and guidelines for the interpretation of sequence variants: a joint consensus recommendation of the American College of Medical Genetics and Genomics and the Association for Molecular Pathology. Genet Med. 2015 May;17(5):405-24.

8. Oza AM, DiStefano MT, Hemphill SE, Cushman BJ, Grant AR, Siegert RK, et al. Expert specification of the ACMG/AMP variant interpretation guidelines for genetic hearing loss. Hum Mutat. 2018 Nov; 39(11):1593-613.

9. Kyei Barffour I, Kyei Baah Kwarkoh R. GREB1L as a candidate gene of Mayer-Rokitansky-Küster-Hauser Syndrome. Eur J Med Genet. 2021 Mar;64(3):104158.

10. Rentzsch P, Schubach M, Shendure J, Kircher M. CADD-Splice-improving genome-wide variant effect prediction using deep learningderived splice scores. Genome Med. 2021 Feb;13(1):31.

11. Ioannidis NM, Rothstein JH, Pejaver V, Middha S, McDonnell SK, Baheti S, et al. REVEL: an ensemble method for predicting the pathogenicity of rare missense variants. Am J Hum Genet. 2016 Oct;99(4):877-85.

12. Karczewski KJ, Francioli LC, Tiao G, Cummings BB, Alföldi J, Wang $\mathrm{Q}$, et al. The mutational constraint spectrum quantified from variation in 141,456 humans. Nature. 2020 May;581(7809):434-43.

13. Jung KS, Hong KW, Jo HY, Choi J, Ban HJ, Cho SB, et al. KRGDB: the large-scale variant database of 1722 Koreans based on whole genome sequencing. Database (Oxford). 2020 Jan;2020:baaa030. 